\title{
How does brief guided mindfulness meditation enhance empathic concern in novice meditators?: A pilot test of the suggestion hypothesis vs. the mindfulness hypothesis
}

\author{
Motohide Miyahara $^{1}$ (1) $\cdot$ Rebekah Wilson $^{2} \cdot$ Tessa Pocock $^{3} \cdot$ Tomoko Kano $^{4} \cdot$ Hiroyuki Fukuhara $^{4}$ \\ Published online: 11 July 2020 \\ (C) Springer Science+Business Media, LLC, part of Springer Nature 2020
}

\begin{abstract}
Despite the widespread popularity of mindfulness meditation for its various benefits, the mechanism underlying the meditation process has rarely been explored. Here, we present two preliminary studies designed to test alternative hypotheses: whether the effect of brief guided mindfulness meditation on empathic concern arises from verbal suggestion (suggestion hypothesis) or as a byproduct of an induced mindfulness state (mindfulness hypothesis). Study 1 was a pilot randomized control trial of sitting (breath-and-body) meditation vs. compassion meditation that provided preliminary support for the mindfulness hypothesis. Study 2 was set up to rule out the possibility that the meditation effects observed in Study 1 were the effects of repeated measures. An inactive control group of participants underwent the repeated measures of empathic concern with no meditation in between. The pre-post comparison demonstrated no significant changes in the measures. Thus, the results of two studies supported the mindfulness hypothesis. Limitations of the present study and future research directions are discussed.
\end{abstract}

Keywords Suggestion $\cdot$ Mindfulness $\cdot$ Meditation $\cdot$ Empathy $\cdot$ Prosocial behavior

\section{Introduction}

Over the past decade, mindfulness training has received unprecedented popularity and widespread application in education (Maynard et al. 2017), health (Creswell 2017), and workplace (Beard 2014) sectors. While an increasing amount of research has been devoted to evaluating the efficacy of mindfulness training in each of these sectors, an early caveat was

Electronic supplementary material The online version of this article (https://doi.org/10.1007/s12144-020-00881-3) contains supplementary material, which is available to authorized users.

Motohide Miyahara

motohide.miyahara@gmail.com

1 Department of Clinical Psychological Science, School of Medicine, Hirosaki University, 66-1, Hon-cho, Hirosaki, Aomori 036-8564, Japan

2 Exercise Medicine Research Institute, Edith Cowan University, 270 Joondalup Drive, Joondalup, WA 6027, Australia

3 School of Nursing, University of Auckland, Building 505, Level 2, 85 Park Road, Grafton, Auckland, New Zealand

4 College of Letters, Ritsumeikan University, 56-1 Tojiin-kita-machi, Kita-ku, Kyoto, Japan issued to pay equal attention to testable theories of possible mechanisms underlying the training effects (Shapiro et al. 2006). The investigation into the potential underlying process prompts a challenge to the fundamental basis of dependent and independent variables for measuring and detecting the training effects (e.g., MacCoon et al. 2012; Davidson and Kaszniak 2015; Quaglia et al. 2016; Kreplin et al. 2018). In line with this critical approach to investigating the mechanisms of mindfulness training, the present study focuses on the effects of brief guided mindfulness meditation to elucidate the contribution of verbal guidance and the altered state of mindfulness.

Mindfulness meditation is a part of the standard mindfulness training curriculum (e.g., Kabat-Zinn 2005; Williams and Penman 2011) which typically introduces students to raisin exercise, body scan, and yoga. Meditation exercises are often scheduled later in the curriculum, starting with instruction on sitting postures, followed by a suggestion that students pay attention to their breath and physical sensations. This stage of the meditation exercise is called sitting meditation (e.g., Kabat-Zinn 2005) or breath-and-body meditation (Williams and Penman 2011). In an advanced stage of the meditation exercises, students are instructed to cultivate their love kindness, empathy, or compassion first towards close people who students relate well to, then towards neutral people (e.g., 
strangers on the street), and even towards least favorite people. This type of meditation is called love kindness [metta], compassion (e.g., Kabat-Zinn 2005), or befriending meditation (Williams and Penman 2011). Thus, there are two types of meditation exercises, sitting and compassion meditation, in mindfulness training.

To understand how novice meditators experience the two types of standard 35 min-long guided mindfulness meditation, Miyahara et al. (2017a) piloted and tested the feasibility of the meditation and measuring its effects on empathic concern, that is, "the ability to empathize with the victims of circumstance and the attitude or readiness to engage in prosocial behavior" (Miyahara et al. 2017b, p. 160), compassionate love, and stress response. With caution in mind that the efficacy data from pilot and feasibility studies were preliminary for hypothesis testing (Lancaster et al. 2004), Miyahara et al. (2017a) analyzed data from the pilot and feasibility study. The pilot trial indicated an increase in empathic concern and compassionate love, and a decrease in stress response in both meditation groups. In contrast, the feasibility study not only found a decrease in stress response, but also a decrease in empathic concern in both meditation groups with a more prominent decline in the sitting meditation group than in the compassion meditation group. The authors attributed the seemingly inconsistent findings between the pilot and feasibility trials in their paper (Miyahara et al. 2017b) to the influence of a monetary reward for research participation offered to participants in the pilot trial, the drowsiness of participants in the feasibility trial who had just had lunch, and a potential Hawthorne effect in both trials. An alternative interpretation for the decline of empathic concern after both types of meditation could be desensitization to avoid compassion fatigue (Miyahara et al. 2017b).

To obtain less biased effects of the two types of guided mindfulness meditation, we planned to double-blind the current study to ensure both participants and the experimenters were unaware of which condition was being tested or the true purpose of the present study (Frey 2018). If a researcher uses an inactive control design, it is impossible to double-blind studies of mindfulness meditation because participants know whether they meditated or not (Davidson and Kaszniak 2015). However, double-blinding is possible in an active control trial if participants in the experimental and control groups believe that they experience the same meditation. Such a double-blind condition was devised for the present study as detailed in the method section. We also offered neither cash nor tangible reward and limited the guided meditation to $8 \mathrm{~min}$ to avoid drowsiness. Thus, the primary objectives of this randomized pilot study were to ensure the integrity of study protocol, data collection formats and questionnaires, and the acceptability of mindfulness meditation intervention.

The effects of brief (4-8 $\mathrm{min}$ ) mindfulness meditation have been investigated for both sitting (breath-and-body) meditation and compassion meditation. Brief sitting meditation was effective in improving mindful awareness (Ridderinkhof et al. 2017), attention (Ridderinkhof et al. 2017; Norris et al. 2018), pain threshold (Reiner et al. 2015), mood, anxiety, and cardiovascular variables (Zeidan et al. 2010), but not on mind reading accuracy or on empathic responding (i.e., compensating for social exclusion) in a Cyberball game (Ridderinkhof et al. 2017). On the other hand, there is only one brief compassion (love kindness) meditation study by Hutcherson et al. (2008) who provided evidence for increased feelings of social connection and positivity towards neutral strangers. Despite these reported benefits of brief mindfulness meditation, the current understanding of the underlying process is limited (Heppner and Shirk 2018). No past research has examined the effects of sitting (breath-and-body) meditation and compassion meditation on empathic concern in order to determine whether altered empathic concern is an effect of verbal suggestion by the guide (suggestion hypothesis) or an effect of mindfulness induced by meditation (mindfulness hypothesis). The present randomized pilot study also aims at conducting preliminary testing of the alternative hypotheses.

The suggestion hypothesis (Shuck 1991) posits that an idea which intrudes the mind is accepted uncritically and actualized reflexively by activating predisposed capability and memory. During guided mindfulness meditation, verbal guides utter suggestions for meditators to follow in which there are "differences in the status or position of the speaker and hearer as these bear on the illocutionary force of the utterance" (Searle 1976, p. 5), one of the 12 classifications of illocutionary acts in the speech-act theory (Searle 1976). If the suggestion hypothesis is true, and if the ideas conveyed by verbal suggestions are accepted and carried out, the participants allocated to the compassion meditation group would listen to the verbal guide for the compassion meditation, accept without reservation the guide's suggestions to be compassionate to all, and rate the post-test measures of their empathic concern and compassionate love as higher than the pretest measures. Such a change in the ratings on empathic concern and compassionate love should not occur in the sitting meditation group who are only told to focus on the breath, posture, and physical sensations. Instead, the sitting meditation group is expected to increase mindfulness only. Because compassion meditation is also a mindfulness meditation (Condon 2019; Kreplin et al. 2018), an increase in mindfulness ratings is also anticipated in the compassion meditation group.

The mindfulness hypothesis postulates that a single brief session of mindfulness meditation increases mindful awareness, enhances meta-awareness of self and others (Ridderinkhof et al. 2017), and thus results in improved empathic concern (Condon 2019). If the mindfulness hypothesis is true, the participants not only in the compassion meditation group, but also those in the sitting meditation group would increase empathic concern as a result of meditation, despite 
the differences in the verbal guides. It is also logical to assume that the post-test measure of mindfulness would be higher than its pre-test measure in both groups.

\section{Study 1}

Study 1 was set to examine the effects of the two types of meditation (sitting meditation and compassion meditation) on mindfulness, empathic concern, and compassionate love in randomized, double-blind, two separate parallel-group, non-placebo-controlled, 1-hour protocols. It was hypothesized that verbal suggestion would be a potential mechanism in enhancing empathic concern (suggestion hypothesis) and would be supported if a significant increase in empathic concern and love kindness were observed only in the compassion meditation group. An alternative mindfulness hypothesis (i.e., a state of mindfulness, that is presumed to be induced by meditation, enhances empathic concern) would be supported if a significant increase in empathic concern, mindfulness, and love kindness were observed in both types of meditation groups.

\section{Method}

\section{Design}

We used a $2 \times 2$ factorial design with repeated measures of mindfulness, empathic concern, and compassionate love. The first independent factor was a between-subject factor (the type of guided mindfulness meditation: sitting vs. compassion), and the second factor was a within-subject factor (pre-test vs. post-test).

\section{Participants}

A total of 32 participants were recruited from a New Zealand university campus via word of mouth, flyers, posters, and discussion in classrooms. They were asked to participate if they:

- were University students (18-30 years old)

- had a total of 1 hour available to participate

- had no or little (up to 6 months) former experience of meditation, and

- had neither neuropsychiatric disorders nor long-term disability (lasting 6 months or more) that stops them from doing everyday things other people could do.

All participants signed an informed consent form approved by a university research ethics committee before participating in the study. No course credit or monetary reimbursement was given to the participants.

\section{Measures}

The Cognitive Affective Mindfulness Scale-Revised (CAMSR) (Feldman et al. 2007) is a 12-item self-report questionnaire on a 4-point Likert scale which measures the quality of mindful approaches to thoughts and emotions among respondents who are not familiar with the terms related to mindfulness. The scale has shown evidence of reliability (Cronbach's alpha: .81-.85), construct (attention, present focus, awareness and acceptance), convergent and discriminant validity with other measures (Feldman et al. 2007), and sensitivity to change as a result of mindfulness-based therapy (Lenze et al. 2014).

The Compassionate Love Scale (CLS) (Sprecher and Fehr 2005) is a 32-item self-report questionnaire which assesses altruistic love for close others and humanity on a 7-point Likert scale. This scale demonstrated high internal consistency (Cronbach's alpha $=.94)$ and concurrent validity with the measures of social support ( $r=0.56$ and $0.27, p<.001)$ (Sprecher and Fehr 2005).

The Empathic Concern for Disability and Accessibility (ECDA) task (Miyahara et al. 2017b) was developed to measure empathy and prosocial attitude by recreating real-life situations. The task consisted of participants viewing 60 slides and responding to them. In each slide, the participant read a text passage which described a person with disability in an environment and a photograph depicting the environment. The environment was illustrated as either accessible or in some way inaccessible for the person with impairment, impacting on their ability to perform a particular task, such as walking across a road. The person depicted in each slide had either mobility or visual impairment, and used a white cane, wheelchair, etc., appropriate to the impairment and situation portrayed. After viewing each situational slide for $6 \mathrm{~s}$, the participant read and answered two consecutive questions on a 4-point Likert scale ( $0=$ not at all to $4=$ very much): 1$)$ "How much do you empathize with this person?"; 2) "How much do you want to help this person?". Preliminary psychometric evaluation was examined on the helping intention item which indicated strong internal consistency $(\alpha=.96)$ and construct validity with a four-factor solution: Factor 1: obvious inaccessible situations; Factor 2: obvious accessible situations; Factor 3: complex inaccessible situations; Factor 4: complex accessible situations (Miyahara et al. 2017b). The responsiveness of the ECDA scale to change has been demonstrated as an effect of mindfulness meditation (Miyahara et al. 2017a) and of the effects of gender, culture, and priming self-construal (Miyahara et al. 2018). Thus, the content validity and sensitivity to change have been ensured.

For the current study, we modified the Likert scale from 4 points to 11 points to be consistent with the method of equalappearing interval for measuring attitude (Thurstone 1928), 
while increasing variability, reliability (Givon and Shapira 1984), and sensitivity (Leung 2011). We also divided the 60 slides into two similar sets of 30 slides (Set A and Set B). Each participant was presented with one set of slides before meditation (either Set A or Set B) and the alternative set of slides after the meditation (See the feasibility study in Miyahara et al. 2017a). Based on our standardization data (Miyahara et al. 2017b), we ensured the equivalence of the two sets of slides. The order of presenting the two sets of slides were counter-balanced within each meditation group (A-B for 8 participants in the sitting and the compassion meditation groups; B-A for 8 participants in the sitting and the compassion meditation groups) to minimize familiarity bias, desensitization, and compassion fatigue (Ashar et al. 2016).

Conventional self-report measures, such as the CAMS-R for mindfulness and the CLS for love kindness (described below), are vulnerable to response bias or prone to the inherent demand that mindfulness efficacy research implicitly expect participants to "fake good" (Levinson et al. 2014). The ECDA is expected to detect such participants" "cooperation" and serve as a "lie" scale (see Crowne and Marlowe 1960) by showing participant's willingness to empathize and help people with impairment in accessible conditions in which empathy and help are not required as much as in inaccessible conditions.

The Toronto Mindfulness Scale (TMS) (Lau et al. 2006) was developed to measure mindfulness state after mindfulness meditation. The scale consists of 13 items to tap into curiosity of current experience ( 6 items) and decentered awareness (i.e., awareness of experience with some distance, or meta awareness) ( 7 items). This scale has been used for manipulation check of brief mindfulness meditation (e.g., Hafenbrack et al. 2019).

\section{Treatment}

For brief guided mindfulness meditation, two types of prerecorded audio stimuli were prepared by slightly modifying the publicly available audio files on the web site http:// franticworld.com/free-meditations-from-mindfulness/ - one for guided sitting (breath and body) meditation and the other for compassion (befriending) meditation (Williams and Penman 2011). The audio guides for both types of meditation started with an instruction for the posture and included guidance for meta-awareness of mind wandering (Levinson et al. 2014) and meta-cognitive control of switching attention (Bishop et al. 2004) back to the breath and the body. The modifications involved deleting the name of meditation (i.e., breath and body, befriending) and adding a bell sound at the beginning and the end. The duration of both audio files were approximately 8 mins.

\section{Procedures}

A pilot randomized control trial was carried out in the following steps. First, the first author (MM) generated a 32 random sequence of meditation types (either sitting or compassion meditation) on a computer. Secondly, a series of numbered icons (1-32) were created for the sequence of audio files, each of which was linked to one of the two types of mindfulness meditation guides. Third, one of the two paid research assistants (RW, TP), who were blind to the allocation of the meditation types embedded in the numbered audio flies, used Qualtrics on a Mac computer and conducted the experiment which consisted of:

1. Informed consent

2. Pre-test measures

2.1. Cognitive Affective Mindfulness Scale-Revised

2.2. Compassionate Love Scale

2.3. Empathic Concern for Disability and Accessibility task $\mathrm{A} / \mathrm{B}$

3. Treatment (either sitting or compassion meditation)

4. Manipulation check by Toronto Mindfulness Scale

5. Post-test measures

5.1. Cognitive Affective Mindfulness Scale-Revised

5.2. Compassionate Love Scale

5.3. Empathic Concern for Disability and Accessibility task B/A

\section{Debriefing interview}

When a participant finished the pre-test and informed a research assistant, the research assistant told the participant that meditation can be done in any comfortable position of preference, such as sitting on the chair, beanbag, floor, or lying down on the yoga mat. The research assistant turned on a dim light, turned off the florescent light, told the participant that the recorded meditation instruction would start and end with a bell sound, and left the experiment room to give privacy after clicking the numbered audio file and hearing the bell sound. It was not possible to verify how participants were responding to the guided mindfulness meditation in situ (i.e., during the intervention) as checking in on them would disrupt the meditation. Instead, the Toronto Mindfulness Scale (Lau et al. 2006) was administered to measure the mindfulness state for manipulation check immediately after the meditation before the post-test. After the post-test, the first author conducted debriefing interviews on the participants' past meditation experiences, how the guided mindfulness meditation was similar or different to their past experiences, and any comments on the research experience.

Prior to analysis, we screened all dependent variables. Normality was tested by two tests which examined different aspects of normality (Yap and Sim 2011). The Shapiro-Wilk test of normality detected that the pre-test measures of the 
Cognitive Affective Mindfulness Scale-Revised and empathy under accessible condition, and the post-test measure of the Cognitive and Affective Mindfulness Scale violated the normality assumption. The Kolmogorov-Smirnov test for normality revealed that the pre-test measure of helping intention under inaccessible condition and all post-test measures except the Compassion Love Scale violated the normality assumption. However, ANOVA is robust to the violation of normality assumption (Harwell et al. 1992). Moreover, Levene's test for homogeneity and Mauchly's test for sphericity showed no significant departure from the assumptions in all dependent variables. Therefore, we proceeded ANOVA with based analysis without transforming the raw data.

\section{Results}

\section{Descriptive Data and Manipulation Check}

All potential research participants, who contacted, made appointments and came to a research lab, read research information, agreed and signed the consent from. The data of Item 11 to 21 in the Compassion Love Scale from the first 13 participants were unavailable due to a technical problem with Qualtrics. The missing data were imputed with the personmean substitution approach (Hawthorne and Elliott 2005), using the mean of Items 1 to 10 . Demographic characteristics of the Study 1 sample in Table 1 showed no significant $(p>.05)$ age or gender difference between the sitting and the compassion meditation groups.

With regard to the acceptability of treatment, Table 2 indicates that the mindfulness state experienced by the participants in the both meditation groups reached equivalent levels of decentering and curiosity similar to the levels of the standardization sample (Lau et al. 2006) who had prior experiences of mindfulness meditation less than 1 year and practiced a 15-min mindfulness meditation session. There was no significant difference between the three groups $(p>.05)$, therefore, both types of mindfulness meditation intervention were considered acceptable. Table 3 shows means and standard deviations of the CAMS-R, the CLS, and the empathy and helping intention scales of the ECDA task before and after the two types of meditation.

\section{Effects of Guided Mindfulness Meditation on Outcome Measures}

\section{Effects on Cognitive and Affective Mindfulness-Revised (CAMS-R)}

There was a significant main effect of meditation time, $F(1$, $30)=6.88, p=.014, \eta^{2}=.187$, but not of meditation type, $F$ $(1,30)=2.03, p=.164$. The meditation main effect was not qualified by an interaction between meditation type and time, $F(1,30)=1.27, p=.269$. This indicates that CAMS-R increases after meditation, regardless of meditation type.

\section{Effects on Compassionate Love Scale (CLS)}

There was a significant main effect of meditation time, $F(1$, $30)=23.025, p<.001, \eta^{2}=.434$, but not of meditation type, $F$ $(1,30)=.401, p=.532$. The time main effect was not qualified by an interaction between meditation type and time, but not of meditation type, $F(1,30)=.401 p=.532$. This indicates that CLS increases after meditation, regardless of meditation type.

\section{Effects on Empathy}

There was a significant main effect of meditation time (pre vs. post meditation) on the empathy scale of the ECDA, $F$ (2, 29) $=7.88, p=.002, \eta^{2}=.35$, but the main effect of meditation type (sitting vs. compassion) and the interaction between meditation time and meditation type were not significant on the empathy scale of the ECDA. Follow-up ANOVAs revealed that the change from pre-test to post-test was significant only for empathy in the inaccessible condition, $F(1,30)=16.15$, $p<.001, \eta^{2}=.35$. Examination of the means indicates that meditation enhanced empathy for people with impairment in the inaccessible condition regardless of the type of meditation.
Table 1 Demographic characteristics of the sample from Study 1 and Study 2

\begin{tabular}{llll}
\hline & \multicolumn{2}{l}{ Study 1} & Study 2 \\
\cline { 2 - 3 } & Sitting meditation group & Compassion meditation group & Inactive control group \\
\hline Gender $(n)$ & & 6 & 5 \\
Males & 6 & 10 & 11 \\
Females & 10 & & \\
Age (years) & & 21.63 & 21.13 \\
$M$ & 22.38 & 2.16 & 1.46 \\
$S D$ & 2.55 & & \\
\hline
\end{tabular}


Table 2 Means and standard divisions of the subscales scores of Toronto Mindfulness Scale in the sitting meditation group, compassion meditation group and the standardization group

\begin{tabular}{|c|c|c|c|}
\hline & Sitting meditation group & Compassion meditation group & Standardization group \\
\hline$n$ & 16 & 16 & 20 \\
\hline \multicolumn{4}{|c|}{ Subscale Curiosity } \\
\hline$M$ & 16.94 & 18.13 & 16.42 \\
\hline$S D$ & 5.74 & 4.86 & 9.42 \\
\hline \multicolumn{4}{|c|}{ Decentering } \\
\hline$M$ & 21.13 & 22.19 & 23.29 \\
\hline$S D$ & 5.54 & 3.23 & 7.81 \\
\hline
\end{tabular}

\section{Effects on Helping Intention}

There was a significant main effect of meditation time on the helping scale of the ECDA, $F(2,29)=7.21, p=.003$, $\eta^{2}=.33$, but the main effect of meditation type or the interaction between meditation time and meditation type was not significant. Follow-up ANOVAs reveal that the significant change from pre-test to post-test was significant for helping intention both under the accessible condition, $F(1,30)=7.20$, $p=.012, \eta^{2}=.19$ and under inaccessible condition, $F(1$, $30)=13.40, p=.001, \eta^{2}=.31$. Examination of the means indicates that meditation decreased helping intention for people with impairment in the accessible condition, and increased helping intention under the inaccessible condition, regardless of the type of meditation.

\section{Discussion}

Study 1 was designed to test whether the effect of guided mindfulness meditation on empathic concern was due to the verbal suggestion for love and kindness (suggestion hypothesis) or due to mindfulness attained through meditation (mindfulness hypothesis), irrespective of the different content of verbal suggestions given in the two types of meditation. The concerted results of increased cognitive and affective mindfulness, compassionate love, and empathy, prosocial intention in both meditation groups provided empirical support for the mindfulness hypothesis over the suggestion hypothesis.

The results concur with the findings by Hafenbrack et al. (2019) who found that brief sitting (breathing) meditation and love kindness meditation increased a variety of employee prosocial behaviors in simulated work contexts. By contrast, our results are partly consistent and partly inconsistent with Ridderinkhof et al.'s (2017) study on the effect of brief sitting meditation. On one hand, our result of increased cognitive and affective mindfulness in the sitting meditation group is in line with the findings by Ridderinkhof et al. (2017) who reported increased mindful attention and awareness after brief sitting mindfulness meditation. On the other hand, our results on the enhanced empathic concern and compassionate love in the sitting meditation group appears to be incongruent with the

Table 3 Mean and standard deviation of the cognitive and affective mindfulness, compassionate love, and empathic concern for disability and accessibility before and after the two types of meditation

\begin{tabular}{|c|c|c|c|c|}
\hline & \multicolumn{2}{|c|}{ Pre-meditation } & \multicolumn{2}{|c|}{ Post-meditation } \\
\hline & $\begin{array}{l}\text { Sitting } \\
\text { meditation } \\
\text { group } \\
n=16 \\
\text { Mean (SD) }\end{array}$ & $\begin{array}{l}\text { Compassion } \\
\text { meditation } \\
\text { group } \\
n=16 \\
\text { Mean (SD) }\end{array}$ & $\begin{array}{l}\text { Sitting } \\
\text { meditation } \\
\text { group } \\
n=16 \\
\text { Mean (SD) }\end{array}$ & $\begin{array}{l}\text { Compassion } \\
\text { meditation } \\
\text { group } \\
n=16 \\
\text { Mean (SD) }\end{array}$ \\
\hline $\begin{array}{l}\text { Cognitive and Affective } \\
\text { Mindfulness }\end{array}$ & $2.81(0.32)$ & $2.72(0.31)$ & $3.06(0.43)$ & $2.82(0.42)$ \\
\hline Compassion Love & $4.51(0.86)$ & $4.47(0.86)$ & $5.17(1.01)$ & $4.80(1.08)$ \\
\hline \multicolumn{5}{|c|}{ Empathic Concern for Disability and Accessibility } \\
\hline \multicolumn{5}{|c|}{ Empathy } \\
\hline Accessible condition & $5.53(1.55)$ & $5.80(1.60)$ & $5.21(1.95)$ & $5.60(1.74)$ \\
\hline Inaccessible condition & $7.23(1.58)$ & $6.59(1.92)$ & $7.90(1.40)$ & $7.23(1.95)$ \\
\hline \multicolumn{5}{|l|}{ Helping intention } \\
\hline Accessible condition & $4.23(2.04)$ & $5.84(1.64)$ & $3.79(2.20)$ & $5.07(1.95)$ \\
\hline Inaccessible condition & $7.05(2.10)$ & $7.31(1.68)$ & $7.87(1.83)$ & $7.93(1.73)$ \\
\hline
\end{tabular}


null finding by Ridderinkhof et al. (2017) on empathy and prosocial behavior. The reason for the seemingly different results may lie in the measures of empathy and prosocial behavior. Ridderinkhof et al. (2017) measured empathy with the Reading the Mind in the Eye (RME) Test (Baron-Cohen et al. 2001) and prosocial behavior with a Cyberball game. The RME test taps into the emotional and mental states of people in the photographs and does not necessarily assess empathic concern towards victims of circumstance, that is, a combination of personal attributes (visual and physical impairments) and inaccessible environments. Moreover, Cabinio et al. (2015) found a stability of the RME Test across different age groups, which suggests the RME test scores might not be sufficiently sensitive or amenable to change. The validity of responses to witnessing someone being excluded in a Cyberball game has also been questioned as measures of empathic response and prosocial behavior because Cyberball is virtual and its measures may have no relation with real life prosocial behavior (Boyes and French 2009). By contrast, the ECDA in the present study assesses the degree of respondents' empathy and the intention to help individuals with impairment in real-life situations. Future research should further examine the effect of sitting meditation on empathy and prosocial behavior in the real world.

It is reasonable that empathic concern and love kindness increased in the compassion meditation group who were directly instructed to be kind and compassionate in the meditation session. However, why did the sitting meditation group, who were only verbally encouraged to focus on the breath and physical sensations, rate empathic concern and love kindness higher after the meditation than before? If they were not influenced by the verbal instruction to focus on the breath and body, what else could affect the compassion of participants in the sitting meditation?

At the time of this writing, we found an in-press article by Hafenbrack et al. (2019) who had demonstrated that state mindfulness, induced by brief sitting meditation and love kindness meditation, had increased self ratings of compassionate responding in a workplace scenario. Whereas empathy mediated the increase in the compassionate response after love kindness meditation, perspective taking mediated the increase in the compassionate response after sitting meditation. By contrast, our results of Study 1 do not differ between the two types of meditation in the effect of increased empathy and helping intention in the inaccessible conditions, thus providing evidence that the novice meditators enhanced perspective taking for disabled people, despite the type of meditation. The possible differences in the mediating factors between Hafenbrack et al.'s (2019) study and our Study 1 may be due to the consistent scenarios employed for assessing both empathy and helping intention in the present study. Digging deeper, we would like to discuss a few more possible mechanisms underlying the influence of mindfulness on empathic concern, including values clarification, cognitive, emotional and behavioral flexibility, social desirability and response bias.

Shapiro et al. (2006) proposed four mechanisms of mindfulness that lead to change and positive outcome, including self-regulation, values clarification, cognitive, emotional and behavioral flexibility, and exposure. Of the four mechanisms, values clarification operates when one observes and reflects on values with greater objectivity, then rediscovers and chooses values that are truer to oneself. If this mechanism is in operation during the sitting (breath and body) meditation with no direct or indirect suggestion for compassion, the participants' deep values (Brown et al. 2007; Grecucci et al. 2015; Lim et al. 2015; Shapiro et al. 2006; van der Velden et al. 2015), such as empathic and prosocial values or values for compassionate love, are clarified. Value clarification leads to value-based decision (Eberth et al. 2019; Zerubavel and Messman-Moore 2015), and further wise and meaningful actions (Baer 2015). For this reason, the meditator would be more willing to help the victims of circumstance after than before the sitting meditation. Furthermore, the mechanism of cognitive, emotional and behavioral flexibility also facilitates adaptive response to the environment (Franquesa et al. 2017), thereby fostering prosocial intention appropriate for the given built environment. Thus, the mechanisms of values clarification, cognitive, emotional and behavioral flexibility may contribute to understanding this noteworthy and unexpected finding.

It is remarkable to note that after meditation the participants in both groups were less willing to help people with impairment in the accessible condition, and more willing to help them under the inaccessible condition. This finding can be interpreted as enhanced insight into appropriate prosocial behavior after meditation. Wen et al. (2013) define insight in terms of problem-solving performance that involves executive functioning. To gain insight and solve a problem, one needs to take an unorthodox perception of a situation and a creative approach to solving the problem (Parker et al. 2015). To make a judgement of when to and when not to help people with impairment, the participants may gain insight as an outcome of critical thinking which includes three metacognitive skills (Dwyer et al. 2012): (1) analyzing the ability of the potential support recipient and the environment; (2) evaluating whether the environmental demand exceeds the ability or not; (3) inferring the potential need of support recipient. The two types of mindfulness meditation appear to have facilitated the usage of these skills and resulted in more appropriate intention for prosocial behavior.

Improved insight through critical thinking has been a target outcome of mindfulness meditation for a long time. For instance, the cultivation of insight into impermanence is encouraged through meditation practice focusing on the body in the classic Buddhist canon, entitled Satipaț̣hāna-sutta (Anālayo 
2015). However, there is surprisingly few studies that have examined the relation between mindfulness meditation and insight through critical thinking. To date, there has been only one cross-sectional study (Noone et al. 2016) which demonstrated a link between dispositional mindfulness and critical thinking. Brown (2015) also pointed out a lack of research that had examined the relation between insight and compassion. The present study may be among the first intervention studies to provide evidence for appropriate empathic concern, or compassion through enhanced insight and critical thinking as an effect of mindfulness meditation. Future research needs to confirm the effects of enhanced insight and critical thinking with each of their specific measures.

Before fully accepting the mindfulness hypothesis, we should consider the limitations of and alternative explanations for Study 1. In addition to the relatively small sample size of the pilot randomized double-blind trial, Study 1 has no inactive control group, but instead has an active control group (i.e., sitting meditation and compassion meditation served as a control for each other). There is a possibility that the same effects may be obtained with no intervention or with a non-mindfulness intervention. For example, Quaglia et al. (2016) warn that social desirability bias and response bias may lead to mindfulness training-related changes on mindfulness scales. MacCoon et al. (2012) further demonstrate that an active control of a non-mindfulness intervention, named Health Enhancement program, has an effect on reducing psychological distress similarly to the effect of a standard Mindfulness Based Stress Reduction program. To investigate the possibility of response bias and estimate non-treatment effect, we planned Study 2.

\section{Study 2}

The purpose of Study 2 was to examine if the same dependent measures as Study 1 would significantly change as a result of repeated measures administered to an inactive control group without mindfulness meditation. For this study we used a pre-post trial design of a nonmindfulness intervention.

\section{Participants}

Right after the completion of Study 1, a total of 16 participants were recruited in the same manner as Study 1 via word of mouth, flyers, posters, and discussion in classrooms, using the same inclusion/exclusion criteria and the ethical procedures, so that the participants would think that they were participating in the same study as Study 1.

\section{Measures}

We used the same Cognitive Affective Mindfulness ScaleRevised (CAMS-R) (Feldman et al. 2007), the Compassionate Love Scale (CLS) (Sprecher and Fehr 2005), and Empathic Concern for Disability and Accessibility (ECDA) task (Miyahara et al. 2017b). As in the case of Study 1, the order of presenting the two sets of the ECDA are counter-balanced (A-B for 8 participants in the sitting and the compassion meditation groups; B-A for 8 participants in the sitting and the compassion meditation groups).

\section{Non-mindfulness Intervention}

After the pre-test, the first author (MM) conducted a debriefing interview on the participant's knowledge and experience of meditation for approximately $8 \mathrm{~min}$. An inactive waiting control was not used because those participants who were interested in meditation might have meditated while waiting.

\section{Procedures}

A pre-post trial was carried out by the first author (MM) by using Qualtrics on the same Mac computer and conducting the experiment, consisting of

1. Informed Consent

2. Pre-test measures

2.1. Cognitive Affective Mindfulness Scale-Revised

2.2. Compassionate Love Scale

2.3. Empathic Concern for Disability and Accessibility task $\mathrm{A} / \mathrm{B}$

3. Non-mindfulness intervention (interview)

4. Post-test measures

4.1. Cognitive Affective Mindfulness Scale-Revised

4.2. Compassionate Love Scale

4.3. Empathic Concern for Disability and Accessibility task $\mathrm{B} / \mathrm{A}$

5. Guided mindfulness meditation (sitting meditation)

Thus, the orders of debriefing interview and guided meditation in Study 1 were swapped in Study 2.

\section{Results}

\section{Descriptive Data}

Demographic characteristics of Study 2 sample in Table 1 show no significant $(p>.05)$ age and gender difference from the Study 1 sample. Table 4 shows the means and standard deviations of the empathy and helping intention scales of the 
Table 4 Mean and standard deviation of the cognitive and affective mindfulness, compassionate love, and empathic concern for disability and accessibility before and after the two types of meditation

\begin{tabular}{|c|c|c|c|c|}
\hline & \multicolumn{2}{|c|}{ Pre non-mindfulness intervention } & \multicolumn{2}{|c|}{ Post non-mindfulness intervention } \\
\hline & Mean (SD) & $n$ & Mean (SD) & $n$ \\
\hline $\begin{array}{l}\text { Cognitive and Affective } \\
\text { Mindfulness }\end{array}$ & $2.82(0.29)$ & 15 & $2.97(0.51)$ & 15 \\
\hline Compassion Love & $5.06(0.77)$ & 16 & $5.13(0.77)$ & 16 \\
\hline \multicolumn{5}{|c|}{ Empathic Concern for Disability and Accessibility } \\
\hline \multicolumn{5}{|l|}{ Empathy } \\
\hline Accessible condition & $5.96(1.41)$ & 16 & $5.56(2.15)$ & 16 \\
\hline Inaccessible condition & $7.49(1.56)$ & 16 & $7.57(1.76)$ & 16 \\
\hline \multicolumn{5}{|l|}{ Helping intention } \\
\hline Accessible condition & $5.28(1.71)$ & 16 & $4.74(1.95)$ & 16 \\
\hline Inaccessible condition & $7.95(1.37)$ & 16 & $7.80(1.79)$ & 16 \\
\hline
\end{tabular}

ECDA, the CAMS-R, and the CLS before and after the nonmindfulness intervention. For unknown reasons, data from the second administration of the CAMS-R were unavailable from Qualtrics for one participant, and a pairwise deletion of CAMS-R data was applied to this participant, leaving a subtotal of 15 datasets for CAMS-R.

\section{Effects of Non-Mindfulness Meditation Intervention on Outcome Measures}

\section{Effects on Empathy}

There was no significant change from pre-test to post-test in empathy both under accessible $(t=.953,95 \%$ CI: -7.340 $19.215, p=.356)$ and inaccessible $(t=-.169,95 \% \mathrm{CI}$ : $-17.006-14.506, p=.868)$ conditions.

\section{Effects on Helping Intention}

There was no significant change from pre-test to post-test in helping intention both under accessible $(t=1.235,95 \% \mathrm{CI}$ : $-5.942-22.317, p=.236)$ and inaccessible $(t=.234,95 \% \mathrm{CI}$ : $-17.700-22.075, p=.818)$ conditions.

\section{Effects on Cognitive and Affective Mindfulness-Revised (CAMS-R)}

There was no significant change in the CAMS-R from pre-test to post test $(t=-.463,95 \% \mathrm{CI}:-.347-.223, p=.650)$.

\section{Effects on Compassionate Love Scale (CLS)}

There was no significant change in the CLS from pre-test to post test $(t=-.459,95 \% \mathrm{CI}:-.377-.243, p=.653)$.

\section{Discussion}

Study 2 examined the effect of a non-mindfulness intervention on empathic concern, cognitive and affective mindfulness, and compassionate love. The results revealed non-significant changes in these variables, thus suggesting that the significant meditation effects obtained in Study 1 were not simply due to the repeated measures, but the effects of guided mindfulness meditation. Coupled with the results of Study 1, the results of Study 2 lend further support for the mindfulness hypothesis that mindfulness induced by the guided meditation altered empathic concern, mindfulness, and compassionate love.

This result echoes the findings of Bayot et al. (2020) who compared the effects of two types of mindfulness training over 8 weeks: the standard mindfulness training (SMT) (KabatZinn 2005) and the ethics-oriented mindfulness training (EMT) which integrated the Buddhist teachings of loving kindness, compassion and common humanity into the SMT. Their findings revealed a significant increase in self-rating measures of mindfulness as a result of both SMT and EMT and no significant interaction between meditation type and time for self-rating measures of empathic concern, as in the case of our Study 1. They also found that the SMT significantly increased self-report measures of compassion, and that the EMT increased self-report measures of tendency to empathize with the feelings and situation of others more than the SMT had done. Bayot et al. (2020) concluded that their findings on the effects of SMT and EMT on empathy were "marginally significant and lacking specificity" (p. 8), attributing this finding to self-report response bias, which included social desirability discussed earlier, the "short" duration of training, and a lack of specific focus on empathy in EMT. It is interesting that even eight-weeks of mindfulness training, comprising 2 hour sessions per week, were considered inadequate, but still resulted in similar findings to our brief guided mindfulness 
meditation study. The present study therefore adds to the literature by partially ruling out the effect of repetitive measures.

While Study 1 and Study 2 results provide evidence for the mindfulness hypothesis, there remain three primary limitations of these studies. One of the limitations of the studies is their small sample sizes and the missing data. Although ANOVA is robust to violation of normality assumption, some dependent variables were not normally distributed. A second limitation is the fact that the non-mindfulness intervention group in Study 2 is not allocated randomly from a single pool sample as part of Study 1 . There may be a possibility that the time lag between Study 1 and Study 2 could influence the participants' interest in and motivation for the study. A third limitation is a possible experimenter expectancy effect (Colman 2015; Kreplin et al. 2018) due to lack of blinding of the single experimenter in Study 2 who knew the purpose and the hypothesis of the study and conducted the debriefing interview as a non-mindfulness intervention. The experimenter's knowledge and expectations may have influenced the participants' response to the repeated measures. However, it is still likely that the participants would have expected and demonstrated no change between pre-test and post-test even if a blinded research assistant had conducted Study 2 . These three limitations could be resolved by a future study based on a large sample size, single/double-blind, three separate parallel-group protocol. If guided meditation is not administered between the pre-test and post-test measures, but at the end of the experiment, the participants would know the absence of meditation between the repeated measures, and therefore, double-blinding of the inactive control is not possible (Davidson and Kaszniak 2015). Directions for future research also include administering the same protocol to intermediate and advanced meditators. If more developed mindfulness coincides with profound empathic concern and love kindness, such a finding would lend further support to the mindfulness hypothesis.

\section{Conclusion}

In support of the mindfulness hypothesis, novice meditators increased compassionate love and situationappropriate empathic concern as a result of an altered state of mindfulness induced by guided mindfulness meditation. Mindfulness meditation seemed to foster deepened insight into, and enhanced critical thinking of values, irrespective of whether the verbal guidance drew attention to the breath and body or love kindness. The same effect was not obtained when a control task of a debriefing interview was conducted on the topic on meditation experience and knowledge in place of meditation, which further supports the mindfulness hypothesis.

\section{Compliance with Ethical Standards}

Conflict of Interest On behalf of all authors, the corresponding author states that there is no conflict of interest.

\section{References}

Anālayo, B. (2015). Compassion in the Āgamas and Nikāyas. Dharma Drum Journal of Buddhist Studies., 16, 1-31.

Ashar, Y. K., Andrews-Hanna, J. R., Yarkoni, T., Sills, J., Halifax, J., Dimidjian, S., \& Wager, T. D. (2016). Effects of compassion meditation on a psychological model of charitable donation. Emotion, 16(5), 691-705.

Baer, R. (2015). Ethics, values, virtues, and character strengths in mindfulness-based interventions: A psychological science perspective. Mindfulness, 6, 956-969. https://doi.org/10.1007/s12671-0150419-2.

Baron-Cohen, S., Wheelwright, S., Hill, J., Raste, Y., \& Plumb, I. (2001). The "Reading the mind in the eyes" test revised version: A study with normal adults, and adults with Asperger syndrome or highfunctioning autism. Journal of Child Psychology and Psychiatry, 42(2), 241-251. https://doi.org/10.1111/1469-7610.00715.

Bayot, M., Vermeulen, N., Kever, A., \& Mikolajczak, M. (2020). Mindfulness and empathy: Differential effects of explicit and implicit Buddhist teachings. Mindfulness, 11(1), 5-17.

Beard, A. (2014). Mindfulness in the age of complexity. Harvard Business Review, 92(3), 68-73.

Bishop, S. R., Lau, M., Shapiro, S., Carlson, L., Anderson, N., Carmody, J., Segal, Z. V., Abbey, S., Speca, M., Velting, D., \& Devins, G. (2004). Mindfulness: A proposed operational definition. Clinical Psychology: Science and Practice, 11(3), 230-241.

Boyes, M., \& French, D. J. (2009). Having a Cyberball: Using a ballthrowing game as an experimental social stressor to examine the relationship between neuroticism and coping. Personality and Individual Differences, 47(5), 396-401.

Brown, K. W. (2015). Mindfulness training to enhance positive functioning. In K. W. Brown, J. D. Creswell, \& R. M. Ryan (Eds.), Handbook of mindfulness: Theory, research, and practice (pp. 311-325). New York: Guilford Press.

Brown, K. W., Ryan, R. M., \& Creswell, J. D. (2007). Mindfulness: Theoretical foundations and evidence for its salutary effects. Psychological Inquiry, 18(4), 211-237.

Cabinio, M., Rossetto, F., Blasi, V., Savazzi, F., Castelli, I., Massaro, D., Valle, A., Nemni, R., Clerici, M., Marchetti, A., \& Baglio, F. (2015). Mind-Reading ability and structural connectivity changes in aging. Frontiers in Psychology, 6, 1808. https://doi.org/10.3389/fpsyg. 2015.01808 .

Colman, A. M. (2015). A dictionary of psychology (4th ed.). Oxford: Oxford University.

Condon, P. (2019). Meditation in context: Factors that facilitate prosocial behavior. Current Opinion in Psychology, 28, 15-19.

Creswell, J. D. (2017). Mindfulness interventions. Annual Review of Psychology, 68(1), 491-516. https://doi.org/10.1146/annurevpsych-042716-051139.

Crowne, D. P., \& Marlowe, D. (1960). A new scale of social desirability independent of psychopathology. Journal of Consulting Psychology, 24(4), 349-354.

Davidson, R. J., \& Kaszniak, A. W. (2015). Conceptual and methodological issues in research on mindfulness and meditation. American Psychologist, 70(7), 581-592.

Dwyer, C., Hogan, M., \& Stewart, I. (2012). An evaluation of argument mapping as a method of enhancing critical thinking performance in 
e-learning environments. Metacognition and Learning, 7(3), 219244.

Eberth, J., Sedlmeier, P., \& Schäfer, T. (2019). PROMISE: A model of insight and equanimity as the key effects of mindfulness meditation. Frontiers in Psychology, 10. https://doi.org/10.3389/fpsyg.2019. 02389.

Feldman, G., Hayes, A., Kumar, S., Greeson, J., \& Laurenceau, J. P. (2007). Mindfulness and emotion regulation: The development and initial validation of the cognitive and affective mindfulness scale-revised (CAMS-R). Journal of Psychopathology and Behavioral Assessment, 29(3), 177-190. https://doi.org/10.1007/ s10862-006-9035-8.

Franquesa, A., Cebolla, A., García-Campayo, J., Demarzo, M., Elices, M., Pascual, J. C., \& Soler, J. (2017). Meditation practice is associated with a values-oriented life: The mediating role of decentering and mindfulness. Mindfulness, 8(5), 1259-1268.

Frey, B. (2018). The SAGE encyclopedia of educational research, measurement, and evaluation (Vol. 1-4). Thousand Oaks: SAGE Publications, Inc.. https://doi.org/10.4135/9781506326139.

Givon, M. M., \& Shapira, Z. (1984). Response to rating scales: A theoretical model and its application to the number of categories problem. Journal of Marketing Research, 21, 410-419.

Grecucci, A., Pappaianni, E., Siugzdaite, R., Theuninck, A., \& Job, R. (2015). Mindful emotion regulation: Exploring the neurocognitive mechanisms behind mindfulness. BioMed Research International, 2015, 670724-670729. https://doi.org/10.1155/2015/670724.

Hafenbrack, A. C., Cameron, L. D., Spreitzer, G. M., Zhang, C., Noval, L. J., \& Shaffakat, S. (2019). Helping people by being in the present: Mindfulness increases prosocial behavior. Organizational Behavior and Human Decision Processes, 159, 21-38. https://doi.org/10. 1016/j.obhdp.2019.08.005

Harwell, M., Rubinstein, E., Hayes, W., \& Olds, C. (1992). Summarizing Monte Carlo results in methodological research: The one- and twofactor fixed effects ANOVA cases. Journal of Educational Statistics, 17(4), 315-339. https://doi.org/10.2307/1165127.

Hawthorne, G., \& Elliott, P. (2005). Imputing cross-sectional missing data: Comparison of common techniques. Australian and New Zealand Journal of Psychiatry, 39(7), 583-590. https://doi.org/10. 1080/j.1440-1614.2005.01630.x.

Heppner, W. L., \& Shirk, S. D. (2018). Mindful moments: A review of brief, low-intensity mindfulness meditation and induced mindful states. Social and Personality Psychology Compass, 12(12), e12424. https://doi.org/10.1111/spc3.12424.

Hutcherson, C. A., Seppala, E. M., \& Gross, J. J. (2008). Lovingkindness meditation increases social connectedness. Emotion, 8(5), 720-724. https://doi.org/10.1037/a0013237.

Kabat-Zinn, J. (2005). Full catastrophe living: Using the wisdom of your body and mind to face stress, pain, and illness: 15th anniversary edition. New York: Delta Trade Paperback/Bantam Dell.

Kreplin, U., Farias, M., \& Brazil, I. A. (2018). The limited prosocial effects of meditation: A systematic review and meta-analysis. Scientific Reports, 8(1), 2403. https://doi.org/10.1038/s41598-01820299-z.

Lancaster, G. A., Dodd, S., \& Williamson, P. R. (2004). Design and analysis of pilot studies: Recommendations for good practice. Journal of Evaluation in Clinical Practice, 10, 307-312.

Lau, M. A., Bishop, S. R., Segal, Z. V., Buis, T., Anderson, N. D., Carlson, L., Shapiro, S., Carmody, J., Abbey, S., \& Devins, G. (2006). The Toronto mindfulness scale: Development and validation. Journal of Clinical Psychology, 62(12), 1445-1467. https:// doi.org/10.1002/jclp.20326.

Lenze, E. J., Hickman, S., Hershey, T., Wendleton, L., Ly, K., Dixon, D., Doré, P., \& Wetherell, J. L. (2014). Mindfulness-based stress reduction for older adults with worry symptoms and co-occurring cognitive dysfunction. International Journal of Geriatric Psychiatry, 29(10), 991-1000. https://doi.org/10.1002/gps.4086.
Leung, S. O. (2011). A comparison of psychometric properties and normality in 4-, 5-, 6-, and 11-point Likert scales. Journal of Social Service Research, 37, 412-421. https://doi.org/10.1080/01488376. 2011.580697.

Levinson, D. B., Stoll, E. L., Kindy, S. D., Merry, H. L., \& Davidson, R. J. (2014). A mind you can count on: Validating breath counting as a behavioral measure of mindfulness. Frontiers in Psychology, 5, 1202. https://doi.org/10.3389/fpsyg.2014.01202.

Lim, D., Condon, P., \& DeSteno, D. (2015). Mindfulness and compassion: An examination of mechanism and scalability. PLoS One, 10(2), e0118221. https://doi.org/10.1371/journal.pone.0118221.

MacCoon, D. G., Imel, Z. E., Rosenkranz, M. A., Sheftel, J. G., Weng, H. Y., Sullivan, J. C., Bonuse, K. A., Stoney, C. M., Salomons, T. V., Davidson, R. J., \& Lutz, A. (2012). The validation of an active control intervention for mindfulness based stress reduction (MBSR). Behaviour Research and Therapy, 50(1), 3-12. https:// doi.org/10.1016/j.brat.2011.10.011.

Maynard, B. R., Solis, M. R., Miller, V. L., \& Brendel, K. E. (2017). Mindfulness-based interventions for improving cognition, academic achievement, behavior, and socioemotional functioning of primary and secondary school students. Campbell Systematic Reviews, 13(s), 1-144. https://doi.org/10.4073/CSR.2017.5.

Miyahara, M., Harada, T., Tanaka, S., Fukuhara, H., Kano, T., Ono, T., \& Sadato, N. (2017a). Mindfulness meditation for future early childhood teachers in Japan. Teaching and Teacher Education, 65, 136144.

Miyahara, M., Sawae, Y., Briggs, H., Wilson, R., Doihata, K., \& Sugiyama, A. (2017b). An inventory to assess empathic concern for disability and accessibility: Development and preliminary psychometric investigation. Journal of Accessibility and Design for All, 7(2), 159-197.

Miyahara, M., Sawae, Y., Wilson, R., Briggs, H., Ishida, J., Doihata, K., \& Sugiyama, A. (2018). An interdependence approach to empathic concern for disability and accessibility: Effects of gender, culture, and priming self-construal in Japan and New Zealand. Journal of Pacific Rim Psychology, 12, E11. https://doi.org/10.1017/prp.2017. 19.

Noone, C., Bunting, B., \& Hogan, M. J. (2016). Does mindfulness enhance critical thinking? Evidence for the mediating effects of executive functioning in the relationship between mindfulness and critical thinking. Frontiers in Psychology, 6, 2043. https://doi.org/10. 3389/fpsyg.2015.02043.

Norris, C. J., Creem, D., Hendler, R., \& Kober, H. (2018). Brief mindfulness meditation improves attention in novices: Evidence from ERPs and moderation by neuroticism. Frontiers in Human Neuroscience, 12, 315. https://doi.org/10.3389/fnhum.2018.00315.

Parker, S. C., Nelson, B. W., Epel, E. S., \& Siegel, D. J. (2015). The science of presence: A central mediator of the interpersonal benefits of mindfulness. In K. W. Brown, J. D. Creswell, \& R. M. Ryan (Eds.), Handbook of mindfulness: Theory, research, and practice. New York: Guilford Press.

Quaglia, J. T., Braun, S. E., Freeman, S. P., McDaniel, M. A., \& Brown, K. W. (2016). Meta-analytic evidence for effects of mindfulness training on dimensions of self-reported dispositional mindfulness. Psychological Assessment, 28(7), 803-818. https://doi.org/10.1037/ pas0000268.

Reiner, K., Granot, M., Soffer, E., \& Lipsitz, J. D. (2015). A brief mindfulness meditation rraining increases pain threshold and accelerates modulation of response to tonic pain in an experimental study. Pain Medicine, 17(4), 628-635. https://doi.org/10.1111/pme.12883.

Ridderinkhof, A., de Bruin, E. I., Brummelman, E., \& Bögels, S. M. (2017). Does mindfulness meditation increase empathy? An experiment. Self and Identity, 16(3), 251-269.

Searle, J. R. (1976). A classification of illocutionary acts. Language in Society, 5(1), 1-23. 
Shapiro, S. L., Charlson, L. E., Astin, J. A., \& Freedman, B. (2006). Mechanisms of mindfulness. Journal of Clinical Psychology, 62(3), 373-386.

Shuck, A. (1991). Suggestion in education. In J. F. Schumaker (Ed.), Human suggestibility: Advances in theory, research, and application (pp. 326-340). New York: Routledge.

Sprecher, S., \& Fehr, B. (2005). Compassionate love for close others and humanity. Journal of Social and Personal Relationships, 22, $629 \mathrm{e} 651$.

Thurstone, L. L. (1928). Attitude can be measured. American Journal of Sociology, 33, 529-554.

van der Velden, A. M., Kuyken, W., Wattar, U., Crane, C., Pallesen, K. J., Dahlgaard, J., Fjorback, L. O., \& Piet, J. (2015). A systematic review of mechanisms of change in mindfulness-based cognitive therapy in the treatment of recurrent major depressive disorder. Clinical Psychology Review, 37, 26-39. https://doi.org/10.1016/j.cpr.2015. 02.001 .

Wen, M., Butler, L. T., \& Koutstaal, W. (2013). Improving insight and non-insight problem solving with brief interventions. British
Journal of Psychology, 104, 97-118. https://doi.org/10.1111/j. 2044-8295.2012.02107.x.

Williams, M., \& Penman, D. (2011). Mindfulness: An eight week plan for finding peace in a frantic world. New York: Rodale Books.

Yap, B. W., \& Sim, C. H. (2011). Comparisons of various types of normality tests. Journal of Statistical Computation and Simulation, 81, 2141-2155. https://doi.org/10.1080/00949655.2010.520163.

Zeidan, F., Johnson, S. K., Gordon, N. S., \& Goolkasian, P. (2010). Effects of brief and sham mindfulness meditation on mood and cardiovascular variables. The Journal of Alternative and Complementary Medicine, 16(8), 867-873. https://doi.org/10. 1089/acm.2009.0321.

Zerubavel, N., \& Messman-Moore, T. L. (2015). Staying present: Incorporating mindfulness into therapy for dissociation. Mindfulness, 6(2), 303-314. https://doi.org/10.1007/s12671-0130261-3.

Publisher's Note Springer Nature remains neutral with regard to jurisdictional claims in published maps and institutional affiliations. 